\title{
Du maintien de la biodiversité en médecine
}

\author{
J.-D. Zeitoun · J.-H. Lefevre
}

(C) Springer-Verlag France 2010

Une crise démographique menace-t-elle les spécialistes des maladies digestives, médecins et chirurgiens ? Le rapport Milon du Sénat notait une chute de $23 \%$ du nombre de chirurgiens généraux entre 2000 et 2007 [1]. Le rapport Domergue, sur les choix des internes de chirurgie, n'était guère plus optimiste [2].

Concernant la gastroentérologie, plusieurs projections démographiques annoncent une baisse sensible du nombre de praticiens sur l'ensemble du territoire à partir de cette année, tant et si bien que la densité pourrait être amputée d'un cinquième d'ici à 2020 [3].

Creux de vague d'une courbe sinusoïdale ou phénomène pérenne? Difficile de répondre.

Une enquête menée récemment auprès de plus de 1500 étudiants en médecine de toute la France s'est intéressée au problème [4]. Seuls $8 \%$ des étudiants interrogés déclaraient envisager une spécialisation en hépatogastroentérologie (HGE) ou en chirurgie digestive. Une imprégnation clinique, via les stages, semblait favoriser une intention de spécialisation. Près d'un quart des étudiants évoquaient la surcharge de travail, notamment en chirurgie, pour justifier leur désintérêt.

Plus inquiétant sans doute, presque deux tiers dénonçaient un manque d'attrait pour les pathologies concernées ou un intérêt supérieur pour une autre spécialité. Ces impressions ont été confirmées par une autre étude portant

\footnotetext{
J.-D. Zeitoun

Service de gastroentérologie, hôpital Henri-Mondor, 51, avenue du Maréchal de Lattre de Tassigny, F-94010 Créteil cedex, France

Service de proctologie médico-interventionnelle, centre hospitalier Diaconesses-Croix-Saint-Simon, Site Diaconesses : 12-18, rue du Sergent-Bauchat, F-75012 Paris, France

J.-H. Lefevre ( $\bowtie)$

Service de chirurgie digestive, hôpital Saint-Antoine,

184, rue du Faubourg-Saint-Antoine,

F-75571 Paris cedex 12, France

e-mail : jeremie.lefevre@sat.aphp.fr
}

sur 1742 étudiants en sixième année d'études (DCEM4), évaluant la vocation pour une spécialisation en chirurgie. Si la chirurgie viscérale était la quatrième spécialité chirurgicale envisagée (parmi les dix), elle était avantdernière en termes de perception de qualité de vie et septième en termes de revenus [5].

Une autre étude portant sur 1780 étudiants en DCEM4, à la veille des épreuves classantes nationales (ECN, anciennement concours de l'internat), révélait des résultats analogues : moins de $2 \%$ des futurs internes désiraient se spécialiser en HGE, classant la spécialité au $15^{\mathrm{e}}$ rang [6].

Les maladies de l'appareil digestif n'intéresseraient-elles donc pas nos futurs collègues ? Trop de cancers, d'alcooliques, de diarrhées et autres maladies « sales » qui détournent les étudiants, captifs d'un enseignement universitaire parfois déséquilibré, faisant la part belle au lupus, à la sarcoïdose, à la maladie de Vaquez et autres pathologies plus « sexy ». Il n'est pas question évidemment de contester ici la pertinence de l'apprentissage de ces maladies, mais simplement de rappeler qu'elles représentent autant d'items différents aux ECN, quand l'ensemble de la proctologie est résumé en un seul (pathologie hémorroïdaire).

Force est de constater par ailleurs que certains de nos collègues d'autres spécialités plus prisées (médecine interne, cardiologie, ophtalmologie, chirurgie plastique pour ne citer qu'elles) se sont avérés de meilleurs communicants que nous, parvenant à transmettre auprès des étudiants une image plus romantique et attirante de leur discipline, alors même que $25 \%$ des étudiants ayant effectué un stage en gastroentérologie n'ont jamais assisté à un examen endoscopique [4].

À nous donc de leur emboîter le pas pour mieux valoriser nos spécialités lors des stages, des cours en amphithéâtre, en conférence d'internat, dans les manuels... et susciter ainsi des futures vocations en gastroentérologie, en proctologie ou en chirurgie digestive.

Pour que la formation médicale et chirurgicale aux spécialités digestives rime désormais avec développement durable. 


\section{Références}

1. Milon A (2008) Accès à des soins de qualité : le cas de la chirurgie. Rapport d'information du Sénat

2. Domergue J, Guidicelli H (2003) La chirurgie française en 2003, les raisons de la crise et les propositions

3. Hecketsweiler P (2002) Medical demography: the case of hepatogastroenterology. Introduction to a debate. Gastroenterol Clin Biol 4:343-54

4. Zeitoun JD, Lefevre J (2009) Enquête nationale auprès des étudiants en médecine sur l'apprentissage de l'hépatogastroentérologie et de la chirurgie digestive et sur leur vision de l'avenir : le passage à l'acte s'annonce difficile. Journées francophones d'hépatogastroentérologie et d'oncologie digestive, mars 2009, Paris. Gastroenterol Clin Biol 33:A4

5. Lefevre JH, Karila L, Kerneis S, Roupret M (2010) Motivations and choices of French medical students who wish to pursue a career in surgery: results of a national survey of 1,742 students in their $6^{\text {th }}$ year. J Chir (Paris) [in press]

6. Lefevre JH, Karila L, Kerneis S, et al (2009) Doctors to be have a lack of interest for general practice. Results from a national Poll in a population of 1,870 undergraduate French medical students concerning speciality choice. Presse Med 3:157-9 